\title{
30 años de estudio sobre ausentismo laboral en Chile: una perspectiva por tipos de empresas
}

\author{
Francisco $\mathbf{R}$ Mesa $\mathbf{M}^{1 \mathbf{a}}$, Ana María Kaempffer $\mathbf{R}^{2}$. \\ Work absenteeism in Chile according \\ to the type of work place
}

\begin{abstract}
Background: The issue of medically justified work absenteeism has a great relevance in Chile at the present moment. Aim: To analyze sick leaves among people working in hospitals, mines, automotive industry and universities. Material and methods: Analysis of 14 thesis and research papers about absenteeism in Chile. The incapacity rate (number of days with sick leave per worker per yea), the frequency rate (number of sick leaves per year per worker) and the severity rate (mean duration of sick leaves) were calculated. The diseases causing the highest rates of absenteeism were also recorded. Results: The mean age of the studied populations was 36 years old and the most common diseases causing absenteeism were respiratory, rheumatologic and trauma. Hospital workers had the highest incapacity rate with 14.3 days of sick leave per worker per year, followed by mining industry with 12 days, automotive industry with 7.1 days and universities with 6 days. Conclusions: In Chile, respiratory diseases are the main cause of sick leaves and hospital workers have the highest incapacity rate (Rev Méd Chile 2004; 132: 1100-8).
\end{abstract}

(Key Words: Absenteeism; Illness days; Psychology, industrial; Sick leave)

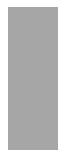

Recibido el 8 de marzo, 2004. Aceptado el 8 de junio, 2004.

${ }^{1}$ Departamento de Gobierno y Empresa, Universidad de los Lagos.

${ }^{2}$ Escuela de Salud Pública, Facultad de Medicina, Universidad de Chile.

angeniero Comercial, Magíster Administración en Salud, Doctor en Ciencias de la Ingeniería.

$\mathrm{E}^{\mathrm{x}}$ xiste un amplio y fuerte debate en nuestra sociedad debido a los posibles cambios a realizar en el sistema de licencias médicas; en medio de la discusión resulta importante recordar quiénes son los financiadores, pagadores y fiscalizadores de cada uno de los diferentes tipos de licencias médicas, los cuales se observan en la Tabla 1.

Como se puede observar, la causa de la licencia médica determina quién será el financiador, el

Correspondencia a: Francisco R Mesa M. Departamento de Gobierno y Empresa, Campus Chinquihue, Universidad de los Lagos. Camino Chinquihue Km. 6, Puerto Montt. Fax: 065-483230. E mail: fmesa@ulagos.cl pagador y el fiscalizador y que no necesariamente resultan ser los mismos organismos.

Los días utilizados en los distintos tipos de licencia médica y el gasto realizado durante 1999 se observan en la Tabla 2.

Al analizar la Tabla 2, se observa que el subsidio por enfermedad común o curativa es el más relevante, debido a la cantidad de días utilizados y por los montos financieros involucrados. Por el contrario, el subsidio pre y postnatal depende de la tasa de fecundidad de las mujeres trabajadoras y en el subsidio por hijo enfermo menor de un año, depende del tipo de enferme- 
dades que tengan los hijos de las mujeres trabajadoras en su primer año de vida.

$\mathrm{Al}$ estudiar la bibliografía internacional sobre ausentismo laboral se sostiene que, aunque el ausentismo laboral se justifica mediante certificados médicos, ello no significa que tales ausencias son exclusivamente por causas médicas ${ }^{3}$, habitualmente el ausentismo laboral es equivalente al ausentismo médicamente justificado por licencias médicas curativas. En dicha bibliografía coexisten cuatro modelos explicativos con diferentes enfoques: económico, psicosocial, médico y retiro organizacional, los que analizaremos a continuación.
1. Modelo económico de ausentismo laboral ${ }^{2}$. Este modelo sostiene que el comportamiento ausentista se debe a la interacción de dos fuerzas: las motivaciones individuales de ausencia de los trabajadores y cuánta ausencia puede ser tolerada por los empleadores, de acuerdo a la tecnología de producción que se utilice. Los trabajadores tienen preferencias individuales de ausencia y como ellos son los actores de la oferta en el mercado de trabajo, eligen la cantidad de ausencias que maximizan sus utilidades mediante el cálculo de los beneficios y costos marginales de las oportunidades que enfrentan. Los empleadores pueden tolerar un cierto nivel de ausencia de los trabajadores, ya

Tabla 1. Tipos, financiadores, pagadores y fiscalizadores de licencias médicas

\begin{tabular}{|c|c|c|c|}
\hline $\begin{array}{l}\text { Tipo de licencia } \\
\text { médica }\end{array}$ & Financiador & $\begin{array}{l}\text { Organismo que realiza la } \\
\text { cancelación }\end{array}$ & $\begin{array}{l}\text { Organismo que efectúa } \\
\text { fiscalización }\end{array}$ \\
\hline $\begin{array}{l}\text { Licencia prenatal y } \\
\text { postnatal }\end{array}$ & $\begin{array}{c}\text { Estado a través } \\
\text { del Fondo Único } \\
\text { de Prestaciones } \\
\text { Familiares } \\
\text { (FUPF) }\end{array}$ & $\begin{array}{c}\text { Empleador } \\
\text { ISAPRE } \\
\text { COMPIN } \\
\text { Cajas de Compensación } \\
\text { INP }\end{array}$ & $\begin{array}{l}\text { ISAPRE } \\
\text { COMPIN }\end{array}$ \\
\hline $\begin{array}{l}\text { Licencia del hijo } \\
\text { enfermo menor de } \\
\text { un año }\end{array}$ & $\begin{array}{c}\text { Estado a través } \\
\text { del Fondo Único } \\
\text { de Prestaciones } \\
\text { Familiares } \\
\text { (FUPF) }\end{array}$ & $\begin{array}{c}\text { Empleador } \\
\text { ISAPRE } \\
\text { COMPIN } \\
\text { Cajas de Compensación } \\
\text { INP }\end{array}$ & $\begin{array}{l}\text { ISAPRE } \\
\text { COMPIN }\end{array}$ \\
\hline $\begin{array}{c}\text { Licencia por } \\
\text { enfermedad } \\
\text { común o curativa }\end{array}$ & $\begin{array}{c}\text { ISAPRE } \\
\text { FONASA }\end{array}$ & $\begin{array}{c}\text { Empleador } \\
\text { ISAPRE } \\
\text { COMPIN } \\
\text { Cajas de Compensación } \\
\text { INP }\end{array}$ & $\begin{array}{l}\text { ISAPRE } \\
\text { COMPIN }\end{array}$ \\
\hline
\end{tabular}

Tabla 2. D ías utilizados y gasto realizado en subsidios de incapacidad laboral 1999

\begin{tabular}{|lcccr|}
\hline Subsidios & $\begin{array}{c}\text { Días utilizados } \\
\text { en subsidios }\end{array}$ & $\begin{array}{c}\text { (\%) } \\
\text { Días }\end{array}$ & $\begin{array}{c}\text { Gasto realizado } \\
\text { (Miles \$) }\end{array}$ & $\begin{array}{r}\text { (\%) } \\
\text { Gasto }\end{array}$ \\
\hline Maternal & 7.037 .914 & $27 \%$ & 50.818 & $22 \%$ \\
Hijo menor de un año & 2.511 .216 & $9 \%$ & 19.822 & $9 \%$ \\
Curativo & 17.051 .751 & $64 \%$ & 163.260 & $69 \%$ \\
Totales & 26.600 .801 & $100 \%$ & 233.900 & $100 \%$ \\
\hline
\end{tabular}

Fuente: Datos obtenidos de Rodríguez y Tokman ${ }^{1}$. 
que al igual que ellos, efectúan el cálculo de los beneficios y costos marginales del ausentismo que perciben, determinando la magnitud de ausencia que minimizan los costos de la empresa, y en consecuencia maximizando las utilidades.

2. Modelo psicosocial del ausentismo laboral ${ }^{2}$. Este modelo sostiene que diferentes culturas de ausencia emergen como resultado de la interacción entre individuos, grupos de trabajo y la organización. El total del tiempo perdido crea una cultura de ausencia en distintas industrias y ocupaciones. La decisión de ausencia se toma en el marco de una cultura de ausencia que puede ser: dependiente, moral, fragmentada o conflictiva. La ausencia es una conducta individual dentro de un contexto social y las motivaciones de ausencia operan restringidas 0 influenciadas por las normas de ausencia propias de las correspondientes culturas de ausencia.

3. Modelo médico del ausentismo laboral ${ }^{3}$. Según este enfoque, existen varios factores que contribuyen a un patrón de ausentismo laboral: demográficos (edad, sexo y nivel ocupacional), satisfacción con el empleo (general, niveles de remuneración, sentido de realización, etc), características organizacionales (a organizaciones y unidades de trabajo más grandes, mayores niveles de ausentismo), contenidos del empleo (niveles de autonomía y responsabilidad) y otros como compromiso, distancia al trabajo, etc.

4. Ausentismo laboral y retiro organizacional ${ }^{4}$. Este modelo sostiene que los trabajadores que se van a retirar voluntariamente de las organizaciones, tienen una mayor tasa de ausentismo que aquellos que permanecen. Existiría una relación positiva entre ausentismo laboral y retiro organizacional. Los trabajadores que abandonan la organización, tienden a ser los más jóvenes y de menor posición que los que permanecen en ella. Estos jóvenes de menor nivel, perciben más oportunidades fuera de la organización que dentro de ella. Los trabajadores de mayor edad y mejor posición tienen mucho más que arriesgar en términos de beneficios sociales y enfrentan menos oportunidades fuera de la organización.

De acuerdo a los modelos presentados se puede concluir que las variables y énfasis en cada uno de ellos son diferentes, pero resulta lógico pensar que el ausentismo laboral médicamente justificado es una interacción de diversos factores y de distintas fuerzas que interactúan entre sí, siendo probable que un modelo más completo y general debería incorporar todas las variables de los modelos ya presentados.

Como se recordará, la mayor importancia de las licencias médicas curativas resulta de los montos totales pagados y del número de días utilizados, sin embargo, no existen en la actualidad estadísticas representativas del ausentismo laboral en las distintas labores y tipos de industrias que componen nuestra economía. Este trabajo es un esfuerzo en tal sentido, ya que en países más desarrollados existen y se realizan estadísticas sectoriales del ausentismo laboral en forma continua y permanente.

\section{MATERIAL y MÉTOdo}

La información básica utilizada en este estudio proviene de 14 tesis y artículos científicos sobre ausentismo laboral por licencias médicas curativas en Chile. En algunos casos, las publicaciones contenían sólo los números absolutos, los cuales fueron elaborados por nosotros para construir las tasas que son habituales en los estudios y análisis de ausentismo laboral ${ }^{5,6}$ : tasa de incapacidad (TI) o número de días de ausencia promedio por trabajador debido a Licencias Médicas Curativas. La tasa de frecuencia (TF) es la cantidad promedio de licencias médicas curativas que ha presentado en un año un trabajador normal; la tasa de severidad (TS) representa la duración promedio de las licencias médicas curativas que fueron presentadas en la población laboral analizada. Los tres indicadores anteriores son complementarios y se deben analizar simultáneamente para lograr comprender las características del ausentismo laboral en una empresa u organización ${ }^{6}$.

Se analizaron los tipos de empresa y población laboral, el porcentaje del sexo femenino, la edad promedio de la población y los meses del año en los cuales hubo mayor presentación de licencias médicas curativas. También se estudiaron los diagnósticos que aparecían en las licencias médicas curativas, los cuales fueron agrupados por los autores según la Clasificación Internacional de Enfermedades (CIE). 
En la clasificación por industrias se resolvió incorporar al Instituto de Salud Pública (ISP) en el sector Universidades y Centros de Investigación. También se resolvió no utilizar la información del estamento administrativo de la Universidad de Chile (UCh) y de la Universidad Metropolitana de las Ciencias de la Educación (UMCE), ya que habrían existido diferentes criterios de clasificación. Además, se decidió no incorporar al estamento Secretaría de la UMCE ya que no existen estamentos comparables en los otros estudios. La limitación principal es el número de población estudiada que sólo alcanzó a 17.232 trabajadores.

\section{Resultados}

Tasas de incapacidad, frecuencia, severidad, promedios por industrias y diagnósticos informados. Es importante recordar que la tasa de incapacidad a nivel nacional durante 1999 fue de 5 días por trabajador, y que esta tasa nacional estuvo en continuo aumento durante la década 1990-99, ya que en 1990 era de sólo 4,3 días, incrementándose en $16 \%$ en sólo 9 años ${ }^{1}$. Sin embargo, algunos autores sostienen que las tasas de ausentismo de nuestro país aún son bajas comparadas con los estándares internacionales ${ }^{7}$.

Los estudios de los que disponíamos fueron agrupados en los diferentes tipos de industrias, ya que esperamos que sectores similares tengan un comportamiento parecido en la utilización de licen- cias médicas curativas. La clasificación nos entregó cuatro tipos de empresas: hospitalaria, minera, industrial y universidades y centros de investigación.

Sector hospitalario. Este sector de servicios es el que presenta la mayor cantidad de estudios, con un total de siete poblaciones distintas en seis estudios realizados. Los datos obtenidos se observan en la Tabla 3.

En la Tabla 3, las tasas más altas de incapacidad (TI) por sobre 19 días promedio, se presentan en: la Clínica Alemana de Santiago ${ }^{8}$, el Hospital San Juan de Dios en Santiago ${ }^{9}$ y el Hospital de Temuco ${ }^{10}$. Un aspecto significativo es que los hospitales con tasas más altas se ubican en grandes ciudades: dos en Santiago ${ }^{8,9}$ y uno en Temuco $^{10}$. Resulta lógico pensar que ciudades más grandes tengan un impacto mayor en el ausentismo, debido a mayores niveles de estrés, mayor violencia y las grandes distancias que deben recorrer los trabajadores, factores que son mencionados en el modelo médico del ausentismo laboral como un factor relevante.

Un caso muy especial lo constituye el trabajo de Jirón ${ }^{9}$ realizado con el personal de Enfermería y Cirugía del Hospital San Juan de Dios, que presenta una alta TI de 34,7 días promedio por trabajador anual, una TF de 4,4 licencias médicas por año y una TS de 7,9 días por licencia médica curativa, no obstante, las características especiales del caso, ya que es una población pequeña de sólo 65 trabajadores, de los cuales $95 \%$ eran mujeres.

Tabla 3. Tasas de incapacidad, frecuencia y severidad en sector hospitalario

\begin{tabular}{|c|c|c|c|c|c|c|c|c|c|c|c|}
\hline Autor & $\begin{array}{l}\text { Año } \\
\text { pública }\end{array}$ & $\begin{array}{c}\text { Población } \\
\text { laboral }\end{array}$ & $\begin{array}{l}\text { Cantidad } \\
\text { LM.C. }\end{array}$ & $\begin{array}{l}\text { Cantidad } \\
\text { días }\end{array}$ & $\begin{array}{l}\text { Tasa } \\
\text { Incap }\end{array}$ & $\begin{array}{l}\text { Tasa } \\
\text { Frec }\end{array}$ & $\begin{array}{l}\text { Tasa } \\
\text { Sev. }\end{array}$ & $\begin{array}{l}\text { Empresa y/0 } \\
\text { ocupación }\end{array}$ & $\begin{array}{l}\% \text { Sexo } \\
\text { femenino }\end{array}$ & $\begin{array}{c}\text { Edad } \\
\text { promedio }\end{array}$ & $\begin{array}{l}\text { Meses } \\
\text { prevalencia }\end{array}$ \\
\hline Gazmuri & 1989 & 790 & 964 & 7.037 & 8,9 & 1,2 & 7,3 & ACHST Salud & $54 \%$ & 33 & \multirow{6}{*}{$\begin{array}{l}\text { Septiembre } \\
\text { Octubre } \\
\text { Julio }\end{array}$} \\
\hline Gazmuri & 1989 & 1.145 & 780 & 5.694 & 5,0 & 0,7 & 7,3 & ACHST Adm & $41 \%$ & 33 & \\
\hline $\begin{array}{l}\text { Silva } \\
\text { Baechler }\end{array}$ & 1989 & 1.187 & 1.471 & 14.620 & 12,3 & 1,2 & 9,9 & Hosp. Temuco & $71 \%$ & 40 & \\
\hline Ibacache & 1996 & 1.173 & 2.557 & 24.160 & 20,6 & 2,7 & 9,4 & Hosp. Temuco & $71 \%$ & 45 & \\
\hline $\begin{array}{l}\text { Silva } \\
\text { Munizaga }\end{array}$ & 1991 & 508 & 595 & 4.740 & 9,3 & 1,2 & 7,9 & $\begin{array}{l}\text { Hosp. } \\
\text { Pta.Arenas }\end{array}$ & $72 \%$ & 37 & \\
\hline Jirón & 1992 & 65 & 285 & 2.256 & 34,7 & 4,4 & 7,9 & Hosp. SJD & $95 \%$ & & \\
\hline Montero & 1998 & 1.957 & 2.538 & 38.915 & 19,8 & 1,3 & 15,3 & \multirow[t]{2}{*}{$\begin{array}{l}\text { Clínica } \\
\text { Alemana }\end{array}$} & $75 \%$ & \multirow[t]{2}{*}{35,2} & \multirow[t]{2}{*}{ Julio } \\
\hline Totales y tic & & 6.825 & 9.190 & 97.422 & 14,3 & 1,3 & 10,6 & & & & \\
\hline
\end{tabular}


Los meses de mayor prevalencia de licencias médicas curativas, resultaron ser los meses de julio, septiembre y octubre.

En resumen, es posible afirmar que los promedios de tasas del sector hospitalario son los más altos de todos los tipos de industrias estudiadas, alcanzando una TI de 14,3 días anuales por trabajador, una TF de 1,3 licencia médica curativa por trabajador anual y una TS de 14,3 días en cada licencia médica curativa.

En relación a los diagnósticos los resultados se observan en la Tabla 4.

Como conclusión, es posible afirmar que, prácticamente en todos los estudios, las enfermedades respiratorias ocupan el primer lugar de prevalencia seguidas de las osteomusculares y digestivas.
Sector minería. En el sector minería existen tres trabajos sobre ausentismo laboral en dos empresas: Minera El Indio ${ }^{14}$ productora de oro en la IV región y División Andina de Codelco Chile ${ }^{15,16}$, productora de cobre ubicada a $80 \mathrm{kms}$ de Santiago (Tabla 5).

Las tasas de ausentismo en esta industria fueron de 11,2 días anuales por trabajador en la TI, 1,3 licencias por trabajador anual en la TF y de 8,4 días por Licencia Médica Curativa en la TS.

En relación a los diagnósticos, éstos se observan en la Tabla 6.

Según Jiménez ${ }^{15}$ un aspecto novedoso es la alta prevalencia de los traumatismos, los cuales podrían relacionarse con la época de los campeonatos deportivos fuera o dentro de la empresa y las actividades deportivas de los trabajadores. Es

Tabla 4. D iagnósticos informados en licencia médicas curativas sector hospitalario

\begin{tabular}{|lrrrrrrrl|}
\hline Autor & Año & Respiratoria & Osteo & Digestivo & Mentales & Traumatismo & Total & Empresa \\
\hline Gazmuri-Salud & $86-87$ & 27,6 & 8,9 & 13,4 & 7,5 & 14,7 & 72,1 & ACHS \\
Gazmuri-Adm & $86-87$ & 28,8 & 8,5 & 15,6 & 8,1 & 14,7 & 75,7 & ACHS \\
Silva-Baechler & 1986 & 26,0 & 10,9 & 10,1 & 8,1 & 8,8 & 63,9 & Hospital Temuco \\
Ibacache & $92-94$ & 31,4 & 22,8 & 10,1 & 15,8 & 7,1 & 87,2 & Hospital Temuco \\
Silva-Munizaga & $86-88$ & 34,6 & 9,4 & 8,1 & 5,2 & 7,9 & 65,2 & Hosp. Pta. Arenas \\
Jirón-Pers Enf Cir & 1991 & 3,0 & 48,0 & 6,5 & 36,1 & S/I & 93,6 & HSJD \\
Montero & 1995 & 29,9 & 14,3 & 13,3 & 6,5 & 7,2 & 71,2 & Hospital Alemán \\
\hline
\end{tabular}

Tabla 5. Tasas de incapacidad, frecuencia y severidad en sector minería

\begin{tabular}{|lcccccccccc|}
\hline Empresa & $\begin{array}{c}\text { Año } \\
\text { pública }\end{array}$ & $\begin{array}{c}\text { Población } \\
\text { laboral }\end{array}$ & $\begin{array}{c}\text { Cantidad } \\
\text { L.M.C. }\end{array}$ & $\begin{array}{c}\text { Cantidad } \\
\text { días }\end{array}$ & $\begin{array}{c}\text { Tasa } \\
\text { Incapac }\end{array}$ & $\begin{array}{c}\text { Tasa } \\
\text { Frec. }\end{array}$ & $\begin{array}{c}\text { Tasa } \\
\text { Sever }\end{array}$ & $\begin{array}{c}\text { Sexo mayor } \\
\text { prevalencia }\end{array}$ & $\begin{array}{c}\text { Edad } \\
\text { promedio }\end{array}$ & $\begin{array}{c}\text { Mes más } \\
\text { prevalencia }\end{array}$ \\
\hline El Indio & 1993 & $4.575^{* *}$ & 7.072 & 63.117 & 13,8 & 1,5 & 8,9 & $0 \%$ Mujeres & 32 & Julio \\
Andina & 1989 & 1.809 & 1.732 & 18.055 & 10,0 & 1,0 & 10,4 & S/I & & Junio \\
Andina & 2001 & 1.193 & 1.634 & 10.475 & 8,7 & 1,3 & 6,4 & S/I & 50 & \\
Totales y Tasas & 7.577 & 10.438 & 91.647 & 12,0 & 1,3 & 8,7 & & & \\
\hline
\end{tabular}

*El dato se obtiene de la tesis original de Cantuarias realizada en la Escuela de Salud Pública de la Universidad de Chile.

Tabla 6. D iagnósticos informados en licencia médicas curativas sector minería

\begin{tabular}{|lcccccc|}
\hline Autor-Empresa & Respiratorias & Osteomuscular & Digestivo & Mentales & Trauma & Total \\
\hline Cantuarias $^{1}$ Minera El Indio & 28,9 & 18,8 & 19,6 & 8,0 & 8,3 & 83,6 \\
Cantuarias $^{2}$ Minera El Indio & 23,8 & 17,5 & 23,9 & 6,1 & 12,2 & 83,5 \\
Jiménez División Andina & 12,1 & 23,8 & S/I & 6,0 & 17,3 & 59,2 \\
Barrientos División Andina & 26,8 & 22,3 & 7,0 & 10,2 & 11,9 & 78,2 \\
\hline
\end{tabular}


posible concluir que en la industria minera el orden de importancia de las enfermedades es como sigue: respiratorias, osteomusculares, digestivas y traumatismos.

Sector industrial. En este sector prácticamente no existen estudios, excepto el trabajo de Oyanguren y cols $^{17}$ era, que entre 1973 y 1980 estudió el ausentismo laboral en la Planta Ensambladora de FIAT de Rancagua, actualmente cerrada. La población en la que se efectuó el estudio era mayoritariamente masculina (97,8\%), joven (32 años, edad promedio) y se desempeñaba en cargos operativos. La Tabla 7 muestra los principales indicadores.

La TI alcanzó a 7,1 días por trabajador, la TF fue 0,6 licencias por trabajador y la TS alcanzó a 12,9 días por licencia médica curativa. En 1974, los diagnósticos que se informaron en el estudio se muestran en la Tabla 8.
Sector universidades y centros de investigación. Los estudios corresponden a: Facultad de Medicina de la Universidad de Chile (UCh) ${ }^{18}$, el segundo en la Universidad Metropolitana de Ciencias de la Educación (UMCE) ${ }^{19}$ y el tercero en el Instituto de Salud Publica (ISP) de Santiago ${ }^{20}$. Se incorporó el ISP dentro del sector universidades ya que su labor normativa, fiscalizadora y de laboratorio de referencia se asimila más a la labor de una Universidad que a cualquiera de los otros tipos de industria. Los resultados se muestran en la Tabla 9.

Como se observa en la Tabla 9, distintos grupos ocupacionales poseen tasas muy diversas entre sí. Así por ejemplo, los académicos poseen bajas TI, que varían entre 5,6 y 6,4 días de LMC anual por académico, una TF que fluctúa entre 0,6 y 0,5 licencias médicas por año y una TS entre 8,8 y 11,7 días por licencia médica. Los auxiliares por su parte, presentan diferencias

Tabla 7. Tasas de incapacidad, frecuencia y severidad en sector industrial

\begin{tabular}{|lcccccccc|}
\hline $\begin{array}{l}\text { Año } \\
\text { publicación }\end{array}$ & $\begin{array}{c}\text { Población } \\
\text { laboral }\end{array}$ & $\begin{array}{c}\text { Cantidad } \\
\text { LM.C. }\end{array}$ & $\begin{array}{c}\text { Cantidad } \\
\text { Días }\end{array}$ & $\begin{array}{c}\text { Tasa } \\
\text { incapacidad }\end{array}$ & $\begin{array}{c}\text { Tasa } \\
\text { frecuencia }\end{array}$ & $\begin{array}{c}\text { Tasa } \\
\text { severidad }\end{array}$ & $\begin{array}{c}\text { Sexo } \\
\text { Preval }\end{array}$ & $\begin{array}{c}\text { Edad } \\
\text { promedio }\end{array}$ \\
\hline 1987 & 849 & 470 & 6.070 & 7,1 & 0,6 & 12,9 & $2,2 \% \mathrm{~F}$ & 32 \\
\hline
\end{tabular}

Tabla 8. D iagnósticos informados en licencia médicas curativas sector industrial

\begin{tabular}{|lcccc|}
\hline Año & Digestivas & Osteomusculares & Mentales & Total \\
\hline 1974 & 28,2 & 24,8 & 24,3 & 89,6 \\
\hline
\end{tabular}

Tabla 9. Tasas de incapacidad, frecuencia y severidad en sector universidades y centros de investigación

\begin{tabular}{|c|c|c|c|c|c|c|c|c|c|c|}
\hline Autor & $\begin{array}{l}\text { Año } \\
\text { Public }\end{array}$ & $\begin{array}{l}\text { Población } \\
\text { laboral }\end{array}$ & $\begin{array}{c}\text { Cantidad } \\
\text { LM.C. }\end{array}$ & $\begin{array}{l}\text { Cantidad } \\
\text { días }\end{array}$ & $\begin{array}{l}\text { Tasa } \\
\text { Incap }\end{array}$ & $\begin{array}{l}\text { Tasa } \\
\text { Frec. }\end{array}$ & $\begin{array}{l}\text { Tasa } \\
\text { Sever }\end{array}$ & $\begin{array}{c}\text { Empresa } \\
\text { ocupación }\end{array}$ & $\begin{array}{l}\text { Sexo } \\
\text { Prev }\end{array}$ & $\begin{array}{c}\text { Edad } \\
\text { promedio }\end{array}$ \\
\hline Corvalán & 1989 & 634 & 398 & 3.520 & 5,6 & 0,6 & 8,8 & $\begin{array}{c}\text { UMCE } \\
\text { Academic }\end{array}$ & $\begin{array}{l}49 \% \\
\text { Mujer }\end{array}$ & 40 \\
\hline Fercovic & 1991 & 390 & 212 & 2.489 & 6,4 & 0,5 & 11,7 & $\begin{array}{c}\text { UCH } \\
\text { Academic }\end{array}$ & $\begin{array}{l}45 \% \\
\text { Mujer }\end{array}$ & \\
\hline Corvalán & 1989 & 231 & 179 & 1.559 & 6,7 & 0,8 & 8,7 & $\begin{array}{l}\text { UMCE } \\
\text { Auxiliar }\end{array}$ & $\begin{array}{l}5,6 \% \\
\text { Mujer }\end{array}$ & 25 \\
\hline Fencovic & 1991 & 145 & 58 & 458 & 3,2 & 0,4 & 7,9 & $\begin{array}{l}\text { UCH } \\
\text { Auxiliar }\end{array}$ & $\begin{array}{l}89 \% \\
\text { Mujer }\end{array}$ & \\
\hline Silva Valero & 1988 & 581 & 634 & 4.288 & 7,4 & 11,1 & 6,8 & $\begin{array}{l}\text { Instituto } \\
\text { Salud Púb. }\end{array}$ & $\begin{array}{l}70 \% \\
\text { Mujer }\end{array}$ & \\
\hline Totales y Tasas & & 1.981 & 1.481 & 12.234 & 6,2 & 0,7 & 8,2 & & & \\
\hline
\end{tabular}


Tabla 10. D iagnósticos informados en licencia médicas curativas sector universidades y centros de investigación

\begin{tabular}{|lcccccccc|}
\hline Autor & Año & Respiratoria & Osteomuscular & Digestivo & Mentales & Trauma & Total & Tipo De \\
\hline Corvalán & $87-89$ & 26,3 & 4,3 & 7,6 & 5,8 & 5,5 & 49,5 & UMCE \\
Fercovic & 1989 & 30,5 & 9,7 & 10,6 & 7,9 & 7,9 & 66,6 & $\begin{array}{c}\text { Académico } \\
\text { UCH, }\end{array}$ \\
Corvalán & $87-89$ & 19,5 & 10,8 & 13,4 & 4,3 & 33,8 & 81,8 & $\begin{array}{c}\text { Académico } \\
\text { UMCE }\end{array}$ \\
Fercovic & 1989 & 27,8 & 11,3 & 14,1 & 9,2 & 7,1 & 69,5 & $\begin{array}{c}\text { Auxiliar } \\
\text { UCH Adm. y }\end{array}$ \\
Silva-Valer & 1987 & 33,7 & 10,6 & 3,4 & 8,1 & 12,9 & 68,7 & $\begin{array}{c}\text { Auxiliares } \\
\text { ISP todas } \\
\text { categorías }\end{array}$ \\
\hline
\end{tabular}

significativas en la TI, ya que varía desde 6,7 días en la UMCE hasta 3,2 días en la UCh. La TF varía entre 0,8 licencias por trabajador al año en la UMCE y 0,4 licencias por trabajador en la UCH. Respecto a la TS, las cifras son relativamente similares, con 8,7 días de duración por licencia médica en la UMCE y 7,9 días de duración por licencia en la UCh. Un posible factor explicativo que puede estar influyendo, es la distinta proporción de mujeres entre ambas universidades, que como consta en el modelo médico, es un importante factor explicativo en el ausentismo laboral médicamente justificado.

Con relación a los diagnósticos, éstos se encuentran agrupados de acuerdo a los diferentes tipos de enfermedades en la Tabla 10.

Como conclusión sectorial de la industria, es posible afirmar que las enfermedades respiratorias ocupan el primer lugar, seguidas de las enfermedades osteomusculares y digestivas en diferente orden de importancia según sea la población en estudio.

\section{DisCUSIÓN Y CONCLUSIONES}

1. Las tasas de ausentismo laboral más altas se encontraron en el sector hospitalario: la tasa de incapacidad fue de 14,3 días por trabajador, la tasa de frecuencia de 1,3 licencia médica curativa anual y la tasa de severidad de 10,6 días en cada licencia médica curativa.

2. En el sector minería la tasa de incapacidad fue de 12 días por trabajador, la tasa de frecuencia de 1,3 licencia médica curativa anual y la tasa de severidad de 8,7 días en cada licencia médica curativa. Este resultado es consistente con la bibliografía internacional que sostiene que la minería es el sector que presenta índices de ausentismo más altos en relación al resto de los sectores industriales ${ }^{5}$.

3. En el sector industrial, la tasa de incapacidad alcanzó a 7,1 días, la tasa de frecuencia a 0,6 licencia médica curativa anual y la tasa de severidad a 12,9 días en cada licencia médica curativa, aunque la muestra es de sólo un estudio.

4. En el sector universidades y centros de investigación, la tasa de incapacidad fue de 6,2 días por trabajador, la tasa de frecuencia de 0,7 licencia médica curativa anual, y la tasa de severidad de 8,2 días en cada licencia médica curativa. También este resultado es consistente con la bibliografía internacional que sostiene que los centros de investigación presentan menores tasas de ausentismo ${ }^{5}$.

5. De los grupos laborales analizados, solamente en seis de ellos aparecen los meses del año con mayor cantidad de licencias médicas, y en 
tres de los casos la mayor cantidad se concentra en el mes de julio.

6. La edad promedio en doce poblaciones laborales estudiadas en los trabajos que poseían dicha información alcanzó 36 años.

7. Es posible afirmar que prácticamente en la totalidad de los estudios analizados, son las

\section{REFERENCIAS}

1. Rodríguez J, Tokman M. Análisis del gasto en subsidios por incapacidad laboral y sus determinantes. Gobierno de Chile. Ministerio de Hacienda. Dirección de Presupuesto. Serie Estudios de Finanzas Públicas. Diciembre 2001. http:// www.dipres.cl/publicaciones/SIL.html

2. KaRL P KAISER. What do we know about employee absence behavior? An interdisciplinary interpretation. Journal of Socio-economics 1998; 27: 79-96.

3. BRINER Rob B. Absence from work (ABC of work related disorders). British Medical Journal 1963; 313: 874 (4).

4. McElroy James C, Paula C Morrow P, James B FENTON. Absenteeism: a comparison of incentives in alternative organizations. Journal of Managerial Issues. Spring 1995; 7: 91(8).

5. TAYLOR PJ. En: Enciclopedia de Salud y Seguridad en el Trabajo. Organización Internacional del Trabajo. Ministerio de Trabajo y Seguridad Industrial. España. 1989 Págs. 5-11. http:// www.mtas.es/insht/EncOIT/Index.htm

6. RicK D HACKETT. Work Attitudes and Employee Absenteeism: A Synthesis of Literature. Journal of occupational psychology 1989; 62: 235-48.

7. Aedo C, Bastías G, Sapew. Subsidios de Incapacidad Laboral en Chile. Fundación Salud y Futuro. Octubre 1999. http://www.saludyfuturo.cl

8. Montero M. Tesis Magíster Salud Pública: Ausentismo Laboral por Licencias Médicas en Instituciones Privadas de Salud: Un caso de la Sociedad de Beneficencia Hospital Alemán-Clínica Alemana. Universidad de Chile. Facultad de Medicina, Escuela de Salud Pública. 1988. http:// catalogo.uchile.cl/web2/tram2.exe/log.ln. enfermedades respiratorias las que ocupan los primeros lugares, hecho importante desde un punto de vista epidemiológico y preventivo. Las enfermedades osteomusculares y traumatológicas presentan altos porcentajes en la mayoría de los trabajos, lo cual es también relevante desde el punto de vista mencionado.

9. Jirón A, Rojas H, Ravera R, Marquéz C. Inasistencia Laboral por Licencias Médicas. Boletín del Hospital San Juan de Dios 1992; 39: 119-24. http:// w w w.hospitalsanjuandedios.cl/ boletin.htm

10. IвACAche J. Tesis Magíster Salud Pública: Ausentismo de causa médica de los funcionarios del Hospital de Temuco años 1992-1993-1994. Universidad de Chile. Facultad de Medicina, Escuela de Salud Pública. 1996. http://catalogo.uchile.cl/ web2/tram2.exe/log.ln.

11. GazmuRi A. Tesis Magíster Salud Pública: Estudio de Ausentismo en trabajadores de un Hospital. Universidad de Chile. Facultad de Medicina, Escuela de Salud Pública. 1989. http://catalogo.uchile.cl/ web2/tram2.exe/log.ln.

12. Silva C, BAEChIER R. Licencia Médicas en personal hospitalario. Rev Méd Chile 1989; 117: 829-33.

13. Siıva MS. Tesis Magíster Salud Pública: Ausentismo Laboral por Morbilidad en el Hospital Regional de Punta Arenas. Universidad de Chile. Facultad de Medicina, Escuela de Salud Pública. 1991. http:// catalogo.uchile.cl/web2/tram2.exe/log.ln.

14. Cantuarias J, Cornejo E. Ausentismo Laboral en una Empresa Minera: Tendencia 1985-1988. Rev Méd Chile 1993; 121: 827-36.

15. JimÉnEz D. Análisis de las Licencias Médicas de trabajadores de CODELCO División Andina como problema de Salud Pública. Boletín Médico del Cobre 1989; 2: 46-51. http://www.bvs.cl/E/ edit.htm

16. Barrientos F, Flores A, Ponce A, Toutin E, ValenZUELA F. Tesis para optar al grado de Magíster en Administración MBA: Análisis del Ausentismo CODELCO Chile División Andina. Universidad de Chile. Facultad de Ciencias Económicas y Admi- 
nistrativas Medicina, Escuela de Salud Pública. 2001. www.facea.uchile.cl

17. Oyanguren $H$, Sandoval $H$, Donoso $H$, Vergara $R$. Estudio de Ausentismo en la Planta de Montaje de FIAT- Rancagua. Chile 1973-1980. Rev Méd Chile 1987; 115: 792-9.

18. Fercovic C. Tesis Magíster Salud Pública: Ausentismo por razones de salud en una Facultad de Medicina y Asociación con algunos hábitos en académicos y estudiantes. Universidad de Chile. Facultad de Medicina, Escuela de Salud Pública. 1991. http:// catalogo.uchile.cl/web2/tram2.exe/log.ln.

19. Corvalán MA. Tesis Magíster Salud Pública: Ausentismo Laboral por causal de salud en la Universidad de Ciencias de la Educación. Universidad de Chile.
Facultad de Medicina, Escuela de Salud Pública. 1989. http://catalogo.uchile.cl/web2/tram2.exe/log.ln.

20. Silva S, Valero J. Tesis Magíster Salud Pública: Estudio Incapacidad Temporal por Morbilidad en una Institución de Salud no asistencial año 1987. Universidad de Chile. Facultad de Medicina, Escuela de Salud Pública. 1988. http://catalogo.uchile.cl/ web2/tram2.exe/log.ln.

\section{Agradecimientos}

Los autores agradecen al Departamento de Gobierno y Empresa de la Universidad de los Lagos, Campus Puerto Montt por el apoyo otorgado a este trabajo. 